\title{
Peripheral capillary nonperfusion and full-field electroretinographic changes in eyes with frosted branch-like appearance retinal vasculitis
}

This article was published in the following Dove Press journal:

Clinical Ophthalmology

15 January 2013

Number of times this article has been viewed

\section{Yoshitsugu Matsui \\ Hideyuki Tsukitome \\ Eriko Uchiyama \\ Yuko Wada \\ Tatsuya Yagi \\ Hisashi Matsubara \\ Mineo Kondo}

Department of Ophthalmology, Mie University Graduate School of Medicine, Tsu, Japan
Correspondence: Mineo Kondo Department of Ophthalmology, Mie University Graduate School of Medicine, 2-I75 Edobashi,

Tsu, 5|4-8507, Japan

Tel $+815923 I 5027$

Fax +8I5 923I 3036

Email mineo@clin.medic.mie-u.ac.jp
Abstract: We report a patient with frosted branch-like appearance retinal vasculitis associated with peripheral capillary nonperfusion and full-field electroretinographic changes. A 62-year-old man presented with sudden bilateral decreased vision accompanied by headaches. His best-corrected visual acuity was 0.01 in both eyes. Fundus examination and fluorescein angiography showed bilateral frosted branch-like appearance retinal vasculitis, and spectraldomain optical coherence tomography showed severe macular edema in both eyes. The cerebrospinal fluid analyses showed an increased lymphocyte count and protein levels. He was treated with systemic corticosteroid therapy, and his best-corrected visual acuity improved to $0.8 \mathrm{OD}$ and $1.0 \mathrm{OS}$ at 6 months after onset. However, fluorescein angiography showed a lack of capillary perfusion in the periphery, and the oscillatory potentials on full-field electroretinography were severely reduced in both eyes. These findings indicated extensive retinal ischemia and inner retinal dysfunction, and that fluorescein angiography and full-field electroretinograms can be useful during follow-up of eyes with frosted branch-like appearance retinal vasculitis.

Keywords: frosted branch angiitis, aseptic meningitis, optical coherence tomography, electroretinogram, oscillatory potentials

\section{Introduction}

Frosted branch angiitis ${ }^{1}$ is a rare type of periphlebitis characterized by white sheathing of the retinal vessels in association with different types of inflammatory eye disease..$^{2-4}$ The onset of frosted branch angiitis is usually sudden, and patients may complain of painless blurred vision, a central blind area, floaters, and photopsias.

Most patients with frosted branch angiitis respond to systemic corticosteroid therapy with good recovery of visual acuity, but various adverse complications have also been reported. These complications include macular scarring, retinal vein or artery occlusion, macular epiretinal membrane formation, diffuse retinal fibrosis, optic disc atrophy, peripheral capillary nonperfusion, and vitreous hemorrhage, all of which have been reviewed elsewhere. ${ }^{3}$

We report our findings in a 62-year-old man with bilateral frosted branch-like appearance retinal vasculitis accompanied by aseptic meningitis. He was treated with systemic corticosteroid therapy with good recovery of visual acuity. However, examinations at 6 months after onset revealed peripheral capillary nonperfusion and severe reduction of the amplitudes of oscillatory potentials on the electroretinogram. These changes suggested extensive retinal ischemia and inner retinal dysfunction, which required photocoagulation. 


\section{Case report}

A 62-year-old man presented with sudden bilateral decrease of vision accompanied by headaches. He did not have any systemic diseases, and his family history revealed no other members with eye disease. He had never experienced oral ulcers or skin lesions previously.

At our initial examination, his best-corrected visual acuity was 0.01 in both eyes, and Goldmann perimetry showed a severe central scotoma in both eyes. The intraocular pressure was $18 \mathrm{mmHg}$ OD and $16 \mathrm{mmHg}$ OS. Slit-lamp examination showed many fine keratic precipitates and cells in the aqueous and vitreous of both eyes. Severe sheathing of the retinal vessels and retinal hemorrhages was detected in both eyes by ophthalmoscopy (Figure 1, upper panel). Fluorescein angiography showed perivenous staining and leakage from the vessels (Figure 1, lower panel). Spectral-domain optical coherence tomography (Spectralis ${ }^{\circledR}$, Heidelberg Engineering, Heidelberg, Germany) demonstrated severe macular edema with central macular thickness of $1045 \mu \mathrm{m}$ OD and $991 \mu \mathrm{m}$ OS (Figure 2, uppermost panel). We also recorded full-field electroretinograms and found that the amplitudes of the mixed rod and cone responses after dark adaptation were severely attenuated in both eyes (Figure 3, middle panel).

On systemic examination, blood count, biochemical analysis including kidney function tests, urine testing, and chest x-rays were within normal limits. Angiotensin-converting enzyme levels were also within normal limits. Tests for syphilis and human immunodeficiency virus were negative.

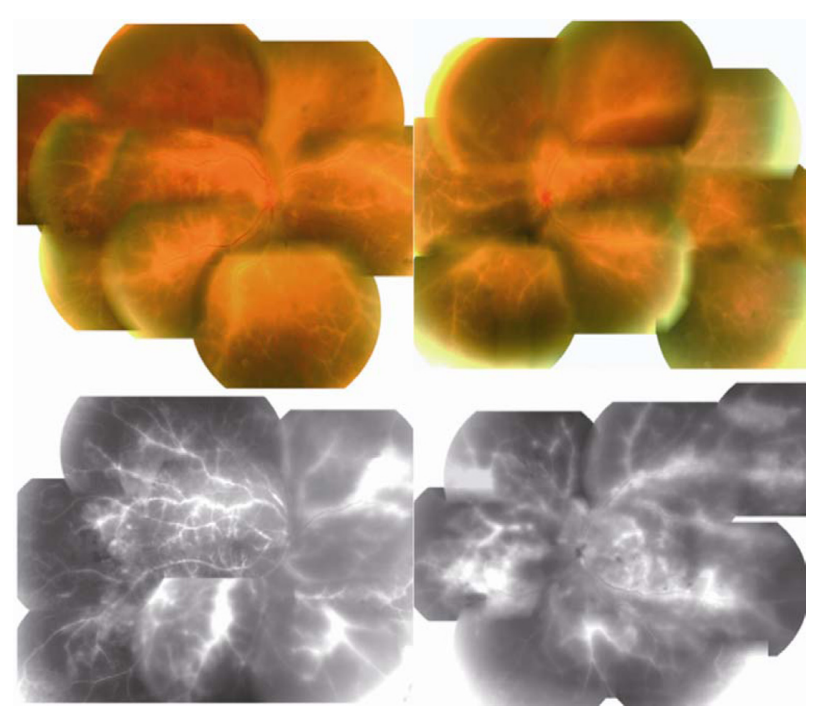

Figure I Fundus photographs and fluorescein angiograms at initial examination showing bilateral diffuse perivascular sheathing and retinal edema with intraretinal hemorrhages (upper panel).

Note: Fluorescein angiography shows extensive vascular leakage and retinal edema (lower panel).

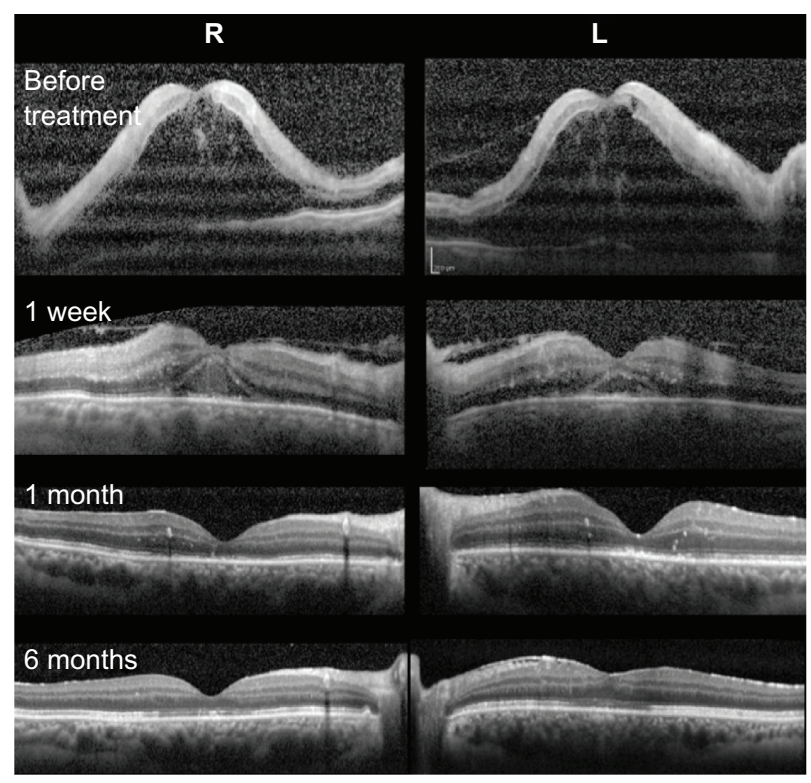

Figure 2 Changes in spectral-domain optical coherence tomograms at onset, and at one week, one month, and 6 months after treatment.

Lupus anticoagulant and anticardiolipin antibody were not detected. The HLA type was A11, A24, B39, B54, DR4, and DR8. Herpes simplex and varicella zoster IgG antibody titers suggested prior exposure only. Polymerase chain reaction analysis of the aqueous humor was negative for herpes simplex, herpes zoster, and cytomegalovirus DNA. Cerebrospinal fluid analysis disclosed an increased leukocyte count $(72 / \mathrm{mL}$, mononuclear cells) and protein level (49 mg/dL; normal 10-40 mg/dL). Cerebrospinal fluid cultures were negative.

Based on the results of these systemic examinations, we diagnosed our patient as having frosted branch-like appearance retinal vasculitis associated with aseptic meningitis. He was treated with pulsed steroid therapy (methylprednisolone $1000 \mathrm{mg} /$ day $\times 3$ days) followed by oral prednisolone $(1 \mathrm{mg} / \mathrm{kg} /$ day), topical steroids, and mydriasis.

One month later, the patient's best-corrected visual acuity improved to 0.6 (OD) and 0.5 (OS), and spectral-domain optical coherence tomography showed a reduction in macular edema (Figure 2, third panel).

Six months after the start of steroid therapy, best-corrected visual acuity was improved to $0.8 \mathrm{OD}$ and $1.0 \mathrm{OS}$ and the fundus had returned to nearly normal (Figure 4 , upper panel). Spectral-domain optical coherence tomography showed that the thickness of the retina was normal but the inner segment/ outer segment junction of the photoreceptors was still disrupted at the fovea in both eyes (Figure 2, lowest panel). We performed fluorescein angiography again, and found that there were extensive areas without capillary perfusion in the 


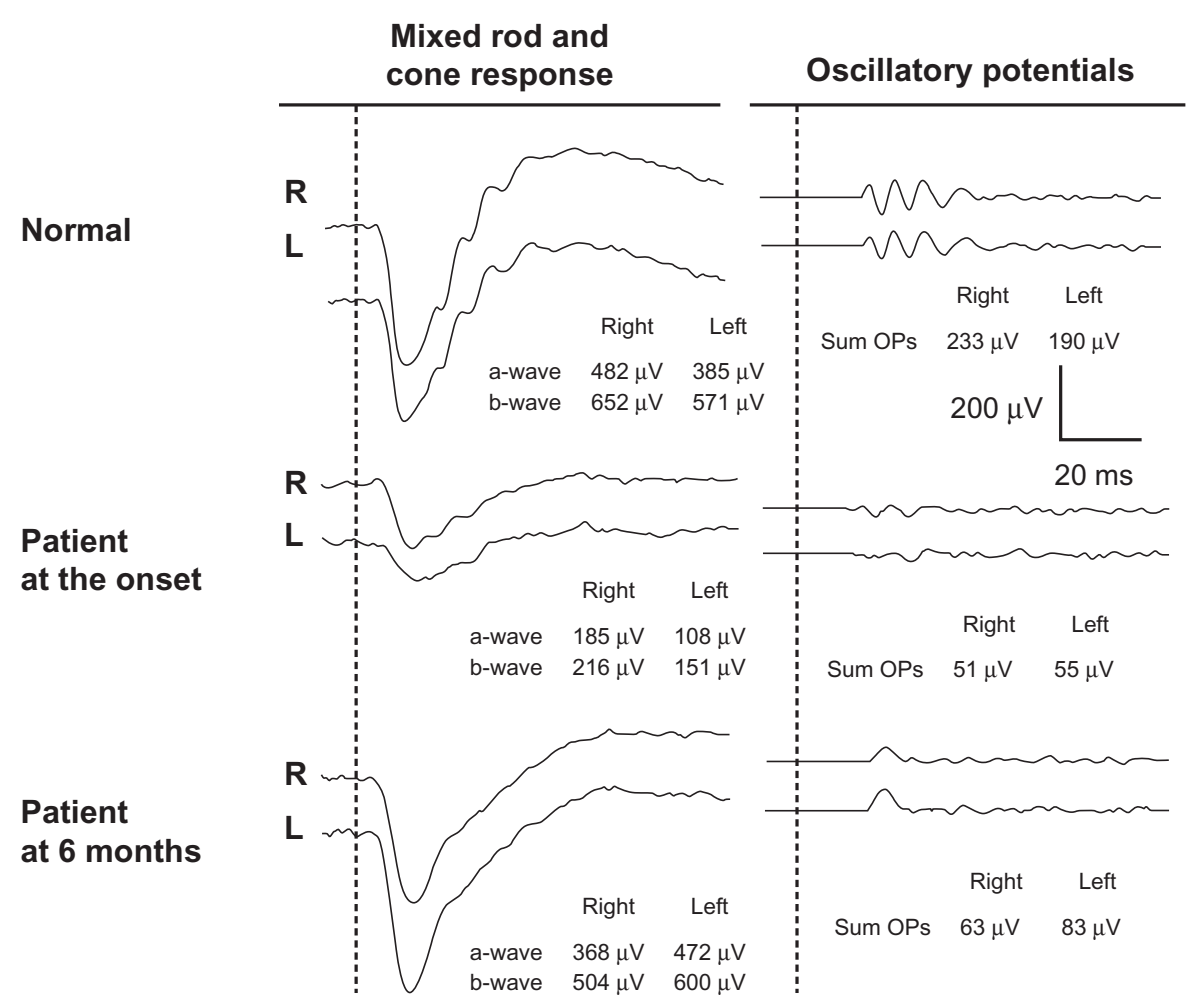

Figure 3 Changes in full-field electroretinograms.

Notes: Full-field mixed rod and cone electroretinograms were recorded after 20 minutes of dark adaptation at onset and 6 months after treatment. Full-field electroretinograms were elicited by a contact lens electrode with a built-in white light emitting diode (LE-2000, Tomey Co, Nagoya, Japan). ${ }^{\prime \prime}$ The stimulus intensity was I0.0 cd-s/m² (photopic units). Although the a-waves and b-waves recovered to normal, the oscillatory potentials were still severely reduced at 6 months after onset.

periphery of both eyes (Figure 4, lower panel). Full-field electroretinograms demonstrated nearly normal a waves and $\mathrm{b}$ waves, but severely reduced oscillatory potentials in both eyes (Figure 3, lowest panel). These results suggested extensive retinal ischemia and inner retinal dysfunction.

We then performed photocoagulation of the peripheral retina to prevent neovascularization and vitreous hemorrhage. There were no retinal complications in our patient during one year of follow-up after photocoagulation.

\section{Discussion}

Frosted branch angiitis is considered to be a clinical subtype of diffuse retinal periphlebitis and is associated with various types of systemic and ocular disease..$^{2-4}$ Frosted branch angiitis is reported to be associated with virus and bacterial infections, lymphoma, leukemia, Crohn's disease, systemic lupus, toxoplasmosis, Behçet's disease, central retinal vein occlusion, nephritis, and other systemic diseases. ${ }^{2-4}$ The frosted branch-like appearance retinal vasculitis in our patient was believed to be associated with aseptic meningitis because he had headaches and an increased lymphocyte count and protein level in his cerebrospinal fluid. Further, no viral infection could be detected.
Johkura et $\mathrm{al}^{5}$ reported a 20-year-old woman with frosted branch angiitis and aseptic meningitis who had headaches, nausea, and vomiting, and cerebrospinal fluid study showed lymphocyte counts increased to $83 / \mathrm{mL}$ and a protein level of $42 \mathrm{mg} / \mathrm{dL}$. More recently, Chaume et $\mathrm{al}^{6}$ reported an 11 -yearold boy with frosted branch angiitis and aseptic meningitis. Their clinical findings and laboratory data were very similar to those in our patient.

In our patient, fluorescein angiography showed extensive areas without capillary perfusion in the peripheral retina and a selective reduction in amplitudes of the oscillatory potentials in both eyes. It is widely accepted that oscillatory potentials originate mainly from inhibitory neural pathways in the inner retina, including those of the amacrine and ganglion cells. ${ }^{7}$ It has also been reported that a selective reduction in the amplitude of oscillatory potentials is also found when inner retinal function is extensively impaired, eg, in diabetic retinopathy or central retinal vein occlusion. ${ }^{7}$ Thus, the findings of a lack of peripheral capillary perfusion and selective loss of oscillatory potentials on the electroretinogram in our patient strongly suggest that the retina was extensively ischemic and required photocoagulation. Luo et $\mathrm{al}^{8}$ also used full-field electroretinograms during follow-up of a 


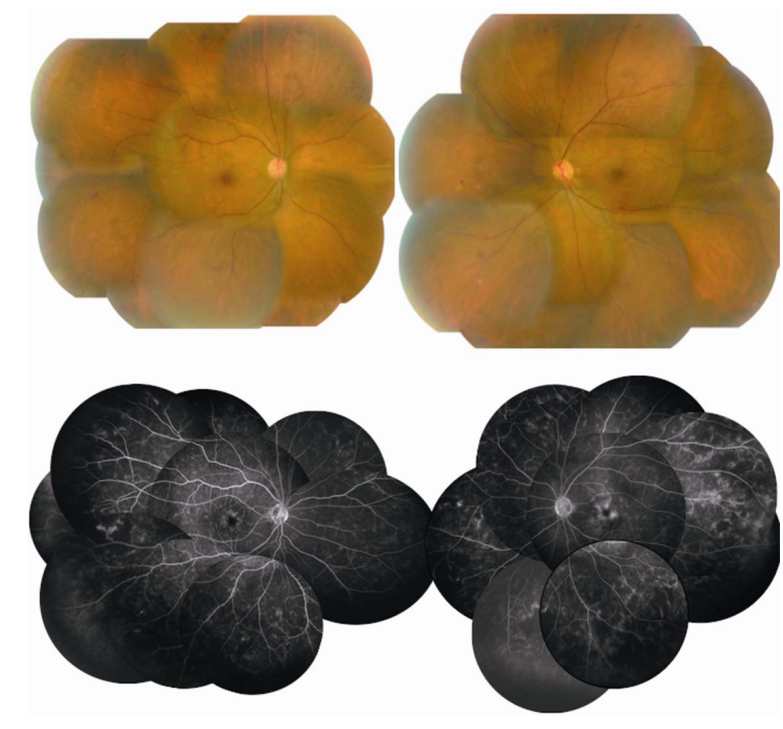

Figure 4 Fundus photographs and fluorescein angiograms 6 months after treatment.

Note: The fundus had recovered to nearly normal, but the fluorescein angiograms show extensive lack of capillary perfusion in the peripheral retina (lower panel).

5-year-boy with frosted branch angiitis, but reported that all electroretinographic components recovered to normal 6 months after treatment.

Our search using PubMed and the Japan Medical Abstracts Society yielded only three papers on frosted branch angiitis associated with peripheral capillary nonperfusion. In 1988, Kleiner et $\mathrm{al}^{2}$ reported a 25 -year-old man with frosted branch angiitis who developed multiple branch vein occlusion, capillary nonperfusion, and retinal neovascularization. In 1989, Terasaki et $\mathrm{al}^{9}$ reported on a 21 -year-old woman with frosted branch angiitis, who developed peripheral capillary nonperfusion and retinal neovascularization which required panretinal photocoagulation 18 months after onset. In 1993, Harigai et $\mathrm{al}^{10}$ reported on a 39-year-old man with frosted branch angiitis who developed peripheral capillary nonperfusion at 7 months after onset. Despite panretinal photocoagulation, vitreous hemorrhages developed in his right eye. Although fluorescein angiography was repeatedly performed during follow-up, full-field electroretinograms were not recorded in these three patients.
The findings in our case suggest that extensive retinal ischemia and inner retinal dysfunction can occur in eyes with frosted branch-like appearance retinal vasculitis, and careful follow-up examinations are needed, even after good recovery of visual acuity. In addition to fluorescein angiography, full-field electroretinography may be useful during follow-up of these patients.

\section{Acknowledgments}

Funding for this work was received from the Ministry of Education, Culture, Science and Technology (23592603), Japan. The authors thank Duco I Hamasaki for editing the manuscript.

\section{Disclosure}

The authors report that they have no competing interests in this work.

\section{References}

1. Ito Y, Nakano M, Kyu N, Takeuchi M. Frosted branch angiitis in a child. Rinsho Ganka. 1976;30(7):797-803. Japanese.

2. Kleiner RC, Kaplan HJ, Shakin JL, Yannuzzi LA, Crosswell HH Jr, McLean WC Jr. Acute frosted retinal periphlebitis. Am J Ophthalmol. 1998;106(1):27-34

3. Walker S, Iguchi A, Jones NP. Frosted branch angiitis: a review. Eye (Lond). 2004;18(5):527-533

4. Kleiner RC. Frosted branch angiitis: clinical syndrome or clinical sign? Retina. 1997;17(5):370-371.

5. Johkura K, Hara A, Hattori T, Hasegawa O, Kuroiwa Y. Frosted branch angitis associated with aseptic meningitis. Eur J Neurol. 2000;7(2):241.

6. Chaume A, Lemelle I, Chastagner P, Angioi K. A case report of frosted branch angiitis associated with aseptic meningitis in a young boy. $\mathrm{J} \mathrm{Fr}$ Ophtalmol. 2011;34(2):129. e1-e5. French.

7. Wachtmeister L. Oscillatory potentials in the retina: what do they reveal. Prog Retin Eye Res. 1998;17(4):485-521

8. Luo G, Yang P, Huang S, Jiang F, Wen F. A case report of frosted branch angiitis and its visual electrophysiology. Doc Ophthalmol. 1998-1999;97(2):135-142.

9. Terasaki H, Yanagida K, Tanaka T. An adult case of frosted branch angiitis with various systemic manifestation. Folia Ophthalmol Jpn. 1989;40(11):2438-2442. Japanese.

10. Harigai R, Seki R, Emi K, Oguro Y, Sato Y. A case of frosted branch angiitis associated with vitreous hemorrhage. Folia Ophthalmol Jpn. 1993;44(6):772-778. Japanese.

11. Kondo M, Piao CH, Tanikawa A, Horiguchi M, Miyake Y. A contact lens electrode with built-in high intensity white light-emitting diodes. Doc Ophthalmol. 2001;102(1):1-9.
Clinical Ophthalmology

\section{Publish your work in this journal}

Clinical Ophthalmology is an international, peer-reviewed journal covering all subspecialties within ophthalmology. Key topics include: Optometry; Visual science; Pharmacology and drug therapy in eye diseases; Basic Sciences; Primary and Secondary eye care; Patient Safety and Quality of Care Improvements. This journal is indexed on

\section{Dovepress}

PubMed Central and CAS, and is the official journal of The Society of Clinical Ophthalmology (SCO). The manuscript management system is completely online and includes a very quick and fair peer-review system, which is all easy to use. Visit http://www.dovepress.com/ testimonials.php to read real quotes from published authors. 